\title{
A Characterization of CS-MRI Reconstruction Using PSO for Random Under Sampling Pattern
}

\author{
G. Shrividya, S.H. Bharathi
}

\begin{abstract}
MR imaging method is widely used for diagnosis applications. The echo signal received from the MR scanning machine is used to generate the image. The data acquisition and reconstruction are the important operations. In this paper the $k$ space is compressively sampled using Radial Sampling pattern for acquiring the k-space data and Particle Swarm Optimization (PSO) with Total Variation (TV) is used as the reconstruction algorithm for the faithful reconstruction of MR image. The experiments are conducted on MR images of Brain, Head Angiogram and Shoulder images. Performance of the proposed method of reconstruction is analyzed for different sampling $k$ space scanning percentages. The reconstruction results are compared with the standard sampling pattern used for compressive sampling prove the novelty of the proposed method. The results are verified in terms of Peak Signal to Noise Ratio (PSNR), Mean Squared Error (MSE) and Structural Similarity index (SSIM).
\end{abstract}

Keywords--- CS-MRI, Random Sampling Pattern, Compressed Sampling, Sampling Trajectory, PSO-TV.

\section{INTRODUCTION}

The novel theory of Compressive Sensing (CS) also called as Compressive Sampling provides basically a new method for data collection [1]. CS demonstrates that the signal or image can be faithfully reconstructed from far few samples than originally required. The theory of CS says that if the image has energy distribution in small portions of pixels or if it is mathematically transformable then, the recovery of a high definition image from very few acquired data is possible. The reconstruction is accomplished with the nonlinear optimization technique which uses the sparsity of the signal than Nyquist sampling theorem which requires more number of samples [2]. Signals or images are sampled at sampling rate of at least double the highest frequency in the signal (Nyquist Rate) in conventional sampling. Huge amount of data is acquired during data acquisition goes waste because; at the stage of compression large portion of the collected data is discarded to facilitate storage and transmission. Collecting only essential samples using appropriate sampling pattern will reduce the scan time [3]. Application of CS in MRI gives potentially significant reduction in scan time which will benefit the patients and health care economics.

A technique called parallel-imaging was developed to accelerate data acquisition process in MRI using arrays of receiver coils with spatially varying sensitivities [4]. Parallel imaging can reconstruct unaliased images from a subset of k-space using Sensitivity Encoding (SENSE)-type algorithms which operate in image space to undo the aliasing, or Generalized Auto calibrating Partially Parallel Acquisitions (GRAPPA)-type algorithms, which operate in $\mathrm{k}$-space to fill in missing points.

Christopher K. et al., [5] proposed a technique called Phase Correction using Conjugate Symmetry reconstruction. $\mathrm{k}$-space has few missing entries due to the phase error. These entries need to be filled for the accurate reconstruction of image.

Missing entries are filled by considering the conjugate symmetry property of k-space. The major objective of the algorithm is the reconstruction of the image by taking input as partial $\mathrm{k}$-space.

Algorithm gives image with phase correction and also gives improved results over the zero filling technique. However it does not perform when many of the entries in kspace are real valued as it is not possible to obtain the conjugate symmetry of real numbers.

K. Miller [6] proposed a technique for partial k-space reconstruction that makes use of an additional function known as weighing function.

It divides the real and imaginary component of the kspace into asymmetric and symmetric components. The major intention of the weighing function is to obtain the identical spreading of spatial frequency in k-space which is generally not uniform. The algorithm takes partial k-space as input and performs image reconstruction. It is similar to phase correction by means of conjugate symmetry algorithm apart from the weighing function factor.

Genetic Algorithm (GA) is global optimization method widely used for solving optimization problems. GA depends on the principles, survival of the fittest theory [10] [11]. Better solutions always evolve from earlier generations until a best possible result is obtained.

Jawad Ali et al. [12] proposed a method for sparse signal recovery based on Hybrid Genetic algorithm. This method uses a chromosome based cross over to produce the off springs with better fitness.

The algorithm is applied for faithful reconstruction of one dimensional sparse signal.

Particle Swarm Optimization (PSO) is the subclass of GA [11]. Development of PSO is based on the social behavior of the groups of population found in nature such as animal herds or schooling of fish or bird flocking [13].

\footnotetext{
Manuscript received September 16, 2019.

G. Shrividya, Research Scholar, School of E\&C Engineering, REVA University \& Faculty, Dept. of E \& C, NMAMIT, Nitte, India.

S.H. Bharathi, School of E\&C Engineering, REVA University, Bengaluru.
} 
Mathematical optimization problem includes maximizing or minimizing an objective function by orderly selecting the input values and calculating the value of the one particular objective function [7]. The input to the optimization process is the objective function or cost function or fitness function and the output is the fitness function of the system. The goal of global optimization is to find the global best value, that is, global maxima or minima of the fitness function.

In this paper we propose that the random sampling of $\mathrm{k}$ space and image recovery through PSO-TV can generate MR image efficiently compared to Cartesian Sampling and PSO-TV. Qualitative and quantitative analysis of the proposed method of CS-MRI reconstruction for different sampling percentages is carried out in terms of PSNR, MSE and SSIM. The efficiency of the proposed method is established in the quality of reconstructed MR image.

This organization of the paper as follows: section II gives the detailed methodology of the proposed method of compressive sensing and image recovery. Section III provides experimental results for the experiments conducted on MR images of Brain, Heart and Shoulder.

\section{METHODOLOGY}

\subsection{MR k-space}

In MRI, image reconstruction is more difficult than other scanning modalities such as CT-scan and Ultrasound scan. Most of the information in k-space resides in the lower frequency region. The higher frequency regions are

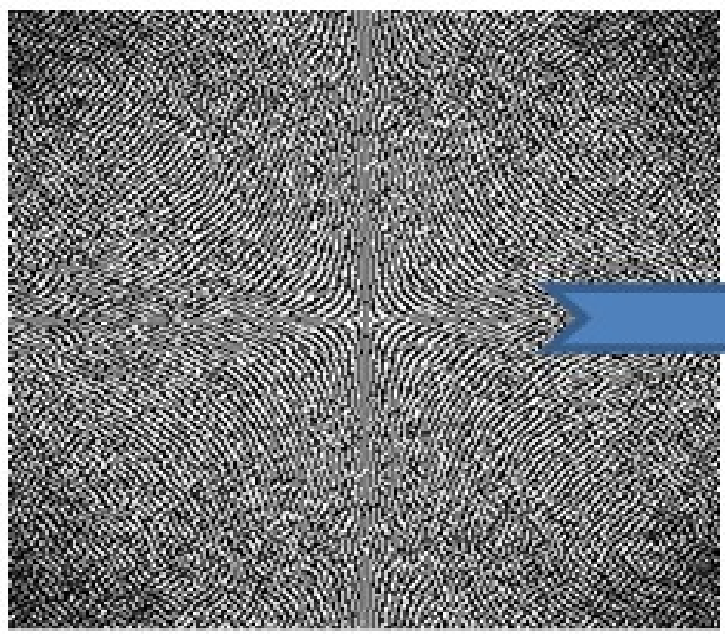

Figure 1: MR k-space and Reconstructed Image

Recovery of an image from its k-space is one of the applications of inverse problems in image or signal processing field [8]. Information regarding the contrast of the image resides in the center region in k-space. The detail of the structure like borders and contour of an image is provided by peripheral regions of $\mathrm{k}$-space.

\section{$2.2 k$-space undersampling patterns}

For the implementation of CS in MRI, patterns with some undersampling are used [9]. The decrease in data which increases the speed of image acquisition is known as undersampling.

More number of samples are collected from the centre of $\mathrm{k}$-space where the energy distribution is more and smaller

normally sparse in k-space, and the majority of the image information is residing near the central part of k-space [15].

The k-space signal is given as,

$$
S(k)=\int_{-\infty}^{\infty} I(x) e^{-i 2 \pi k \Delta k x} d x,-N \leq k \leq N(1)
$$

where the image is $I(x)$ and the image size is $2 N+1$ pixel.The k-space data is related to image data by Fourier Transform. There are three autonomous working coils that give gradient magnetic field along $\mathrm{X}, \mathrm{Y}$ and $\mathrm{Z}$ directions. More precisely, the gradient can be characterized as vector in 3 dimensions. The Larmor frequency $\omega$ indicates the angular momentum of precession generated by magnetic moment of the protons and can be expressed as:

$$
\omega=\gamma B(2)
$$

where $B$ is the strength of the static magnetic field and $\gamma$ is the gyro-magnetic ratio.

The k-space contains the density of NMR signals generated from the patient's body. The signal gets collected on the MRI radio frequency coil when the magnetic field passes through the object.The greater part of the k-space data reside in the lower frequency region shown in Figure 1. The higher frequency regions are generally sparse in k-space and majority of the information of an image is available near the central region of k-space. The FT of a real signal has a conjugate symmetry. Because of this reason the k-space data also follows conjugate symmetry. The high spatial frequencies correspond to the region of an image where the rate of change in intensity is more. The low spatial frequency corresponds to the region of an image where the rate of change in intensity is less.

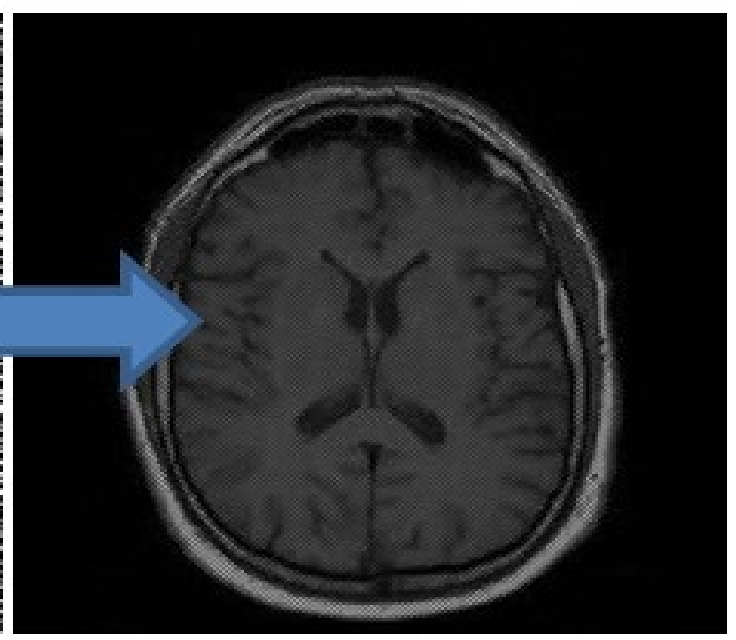

number of samples is collected in the peripheral region of $\mathrm{k}$ space.

As a result, low frequency aliasing were reduced and the performance of reconstruction is improved. The performance of CS reconstruction is improved further by densely sampling certain region around the centre of k-space (low frequency region).

Undersampling patterns such as random, Cartesian and radial are developed in such a way that it acquires more data at the centre than the periphery, as majority of MR image information are at the centre region of $\mathrm{k}$-space. 
Higher sampling density at the centre of k-space contributes to a better Signal to Noise Ratio (SNR) and a lower Mean Squared Error (MSE).

In radial undersampling the lines or the spokes are as shown in Figure 2(a), naturally leads to a denser sampling of information at the centre of the k-space with reduced sampling density towards the periphery [3]. Each line has a unique angle of $\Pi / \mathrm{L}$, where $\mathrm{L}$ is the number of lines.

In Cartesian Grid the k-space lines are the parallel and equispaced to sample Fourier samples. To achieve undersampling using Cartesian pattern fewer lines are selected near the periphery and closer lines near the center of k-space as shown in Figure 2(b). Sampling density near
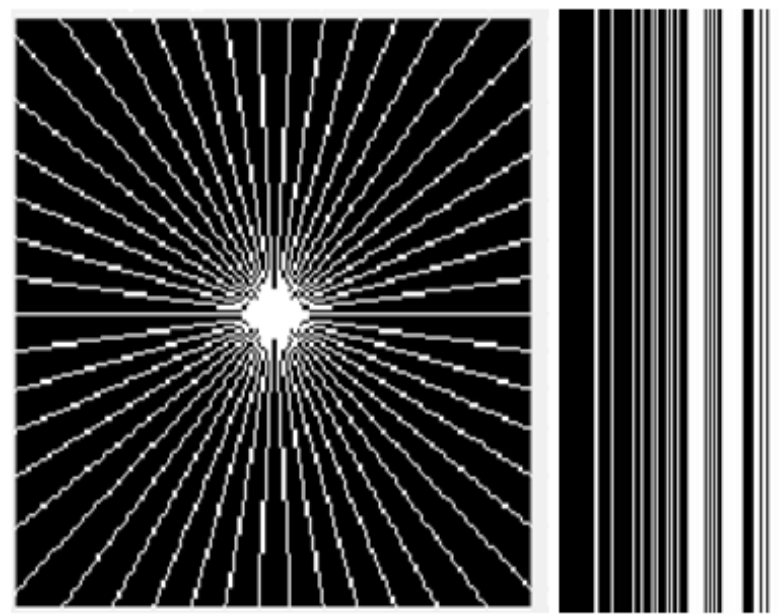

(a) (b) (c)

Figure 2: Undersampling Patterns

\subsection{CS - MRI Reconstruction}

Recovery is achievable under two conditions [3]. The first condition is sparsity which relates to the signal of interest. Sparsity deals with the notion that the information rate of signals can be much lesser than suggested by the bandwidth of the signal.

The second condition is incoherence which relates how the signal is sensed or sampled. The CS uses the fact that lots of ordinary signals are compressible or sparse; it means they have brief representation when expressed in the proper basis $\Psi$.

Incoherence deals with the notion that if the signal or image is sparse in $\Psi$ then it must be spread out in the domain in which they are obtained. This enables faster measurements and uses less memory. Energy distribution in MRI is concentrated more in the lower frequency region than the higher frequency region of k-space.

The general form of data acquirement with incomplete samples for CS-MRI is given as:

$$
b=R x(3)
$$

where $x$ is the reconstructed image, $b$ is the acquired $\mathrm{k}$ space measurement and $R$ is the undersampled Fourier Transform operator which directly relies on the k-space undersampling scheme. Let $\Psi$ denote the linear operator that transforms the data from pixel depiction into the selected
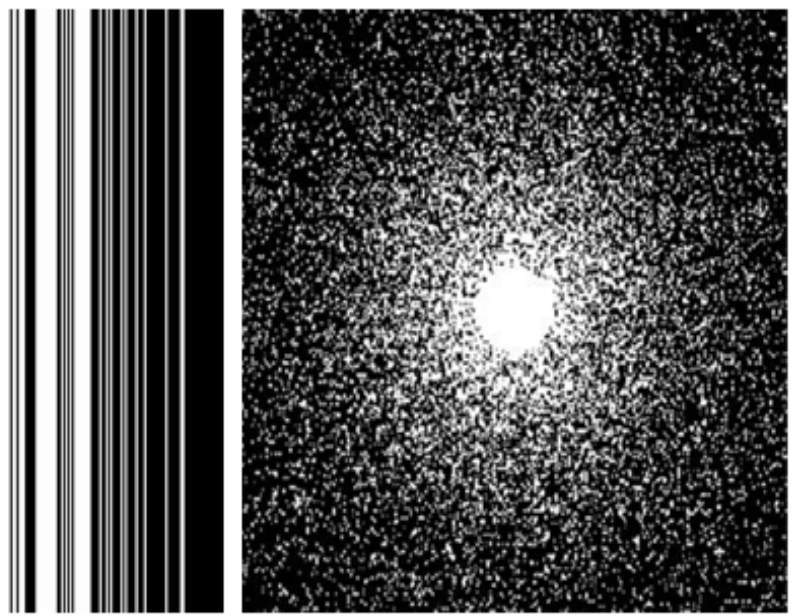

the $\mathrm{k}$-space centre is high and it decreases towards k-space edges. Figure 2(c) shows the random undersampling pattern with dense sampling near the $\mathrm{k}$-space centre. It uses a radial distance calculation between the origin of the $\mathrm{k}$ space and any particular point. By varying this distance different sampling percentage can be obtained at the fully sampled region of the k-space.

\section{(a) Radial (b) Cartesian (c) Random}

depiction. $x$ can be correctly reconstructed from portion of $\mathrm{k}$ space by evaluating the minimization problem which is given as [3]:

minimize $\|\Psi x\|_{1}$ such that $\|R x-b\|_{2}<\epsilon(4)$

where $\epsilon$ is a statistic that describes the magnitude of the error defined as the noise variance or the maximum allowable error in the approximation. Here the $l_{1}$ norm $\|x\|_{1}=\sum_{i}\left|x_{i}\right|$. Minimizing the objective function $\|\Psi x\|_{1}$ promotes the sparsity of the images.

\subsection{Particle Swarm Optimization}

Particle Swarm Optimization (PSO) is one of the most well-known nature inspired metaheuristic optimization algorithms introduced by James Kennedy and Russell Eberhart in 1995 [11]. PSO is based on social activities of groups of population in nature. Usually, a set of animals that have no leaders will discover food by random, go behind a member of the set that has the nearest location with a food source.

The group achieves their best state simultaneously all the way through communication between members of the set who already have a better condition. Member of the set which has a closest distance will update it to its group and the others will progress simultaneously to that position. This procedure would occur repetitively until the optimal conditions or a food source has been discovered.

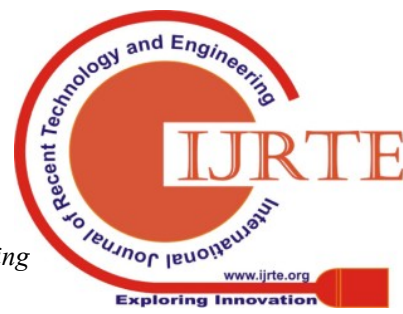




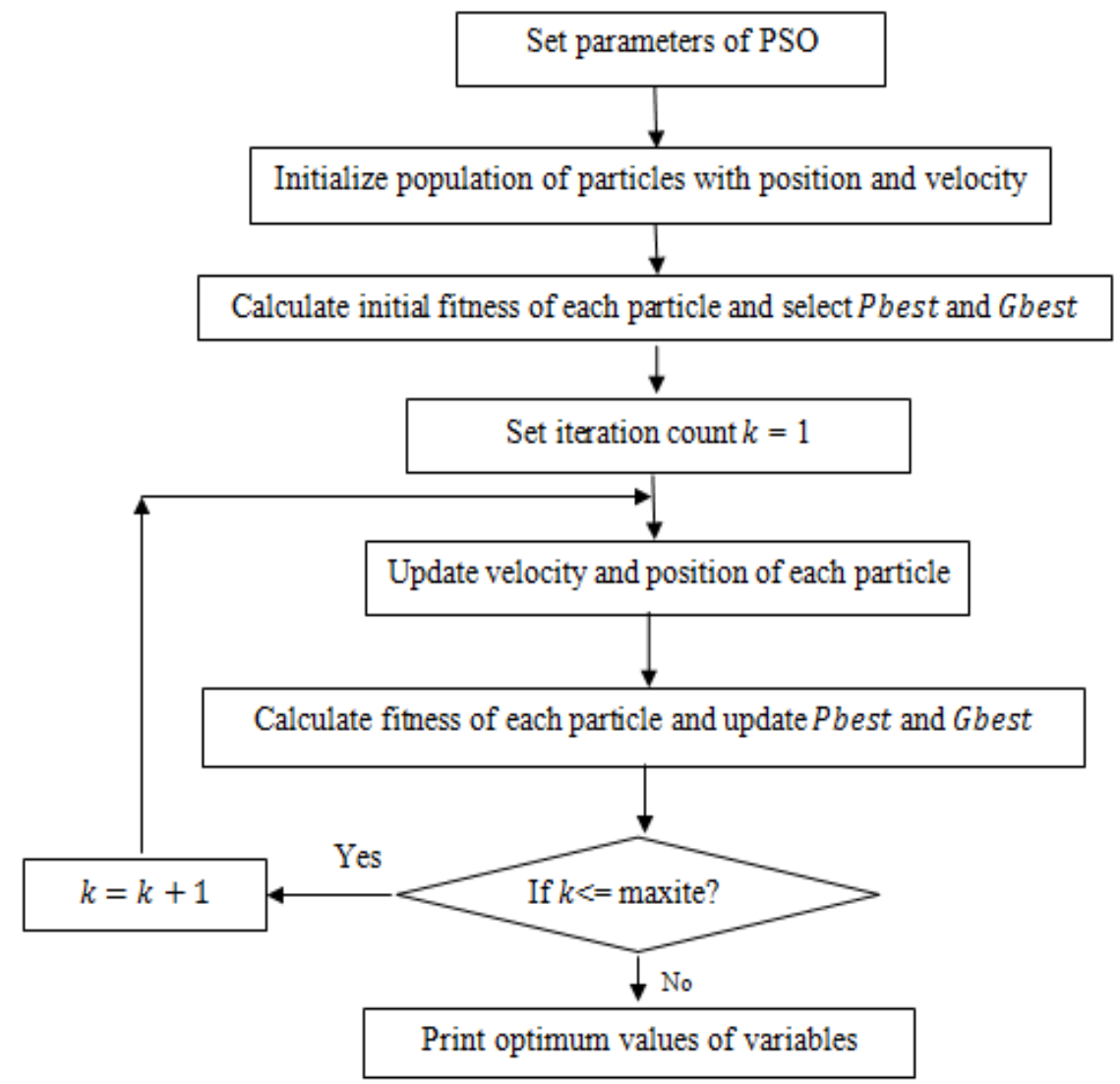

Figure 3: The flowchart of PSO

The objective of the algorithm is to find the optima for every particle. This is done by initially assigning random positions and small random velocities to every particle present in the search space. This algorithm is executed by updating each particle's position based on its velocity, the global best position in the search space and the particle's best position. After each position update, the fitness function is calculated. Over the time, through the combination of searching and utilization of known best positions in the search space, the particles meet together around optima, or several optima.

The flow chart of the algorithm is as shown in Figure 3. The algorithm consists of individual experience, overall experience and the current motion of the particles to choose their next positions in the exploration space. The velocity update of every particle depends on the distance that particle is from the best position found by the swarm, called the global best position (Gbest) and the distance that particle is from its own best position (Pbest), defined as the best solution that the particle has personally come across during its lifetime. Every particle moves with a velocity in the search space.

\subsection{Reconstruction using PSO-TV}

The reconstruction of an undersampled MR image using PSO at lower sampling percentages still leads to artifacts which degrade the quality of reconstructed image. To overcome this problem, Total Variation (TV) minimization model is used with PSO. The fundamental scheme of TV minimization is to restore the ill-conditioned problem with close by well-conditioned problem whose solution approximates the necessary solution [14]. TV minimization also known as TV regularization is the process that has application in noise removal. Higher the noise in a signal means higher is the total variation. Using TV minimization with $\mathrm{PSO}, \mathrm{CS}$ reconstructs the MR image $x$ from undersampled Fourier measurements $b$ by minimizing the objective, which is formulated as

$$
\hat{x}=\arg \min \left\{\frac{1}{2}\|R x-b\|^{2}+\lambda\|x\|_{T V}\right\}(5)
$$

where $\lambda$ is the regularization parameter, $R$ is the Fourier Transform of $x$. The data fidelity is represented by the first term, where the distance between the measured k-space and the expected k-space is minimized. Second term is generally added to the objective to suppress the noise. $\|x\|_{T V}$ represents TV of solution of $x$.

\section{EXPERIMENTAL RESULTS AND DISCUSSIONS}

The experiments are conducted on MR images of brain, head angiogram and shoulder. The images are obtained from MR_tip data base. The random undersampling pattern is used to compressively sample the $\mathrm{k}$-space with different sampling percentages.

The three practical MR images selected for experiments have fine differences in their structure throughout. Figure 4.showsrandom sampling pattern and the reconstructed MR image with different sampling percentages using random undersampling pattern for MRI of brain, head angiogram and shoulder respectively. The undersampling process leads to artefacts at lower sampling percentages. It is observed that the effect of artefacts is less with random sampling pattern. 

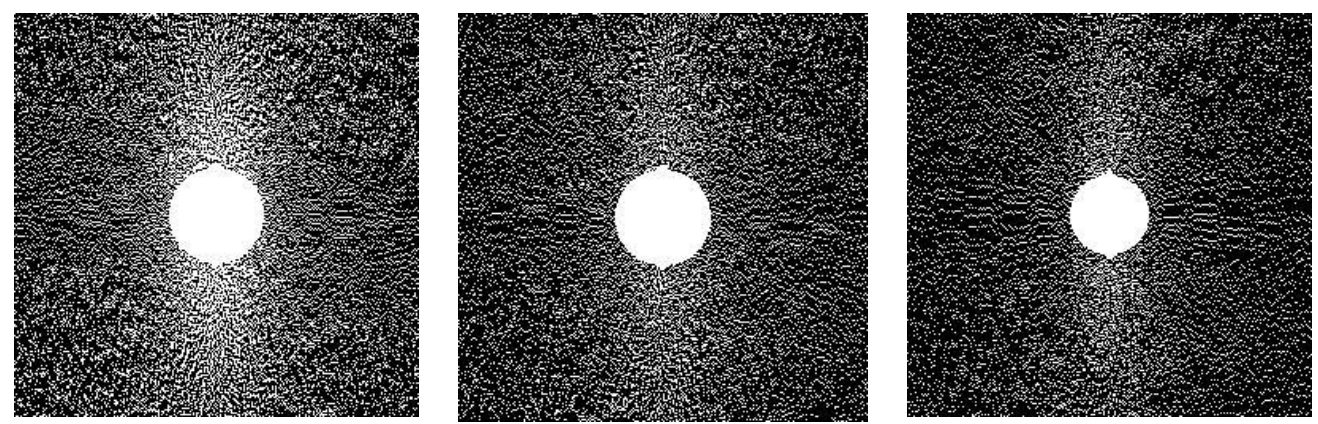

Brain
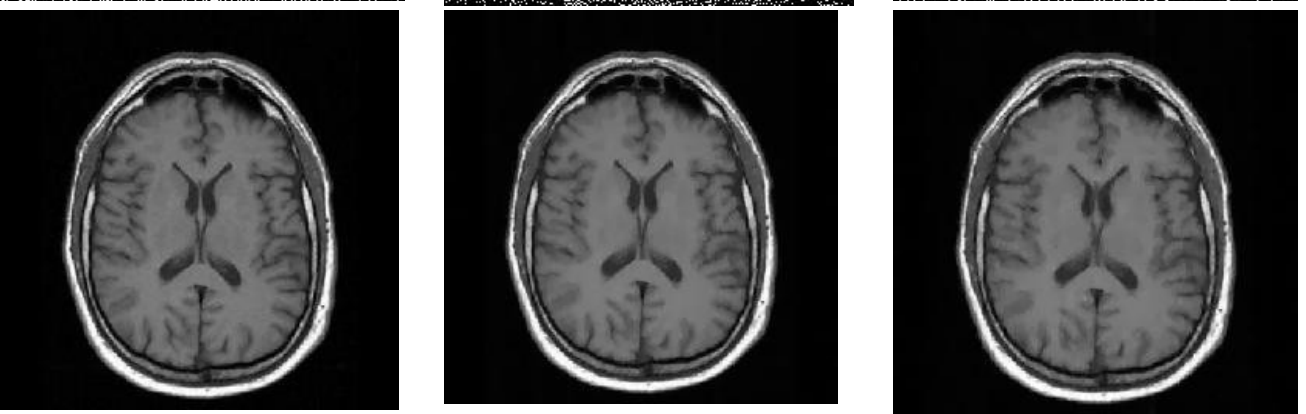

Head

Angiogram
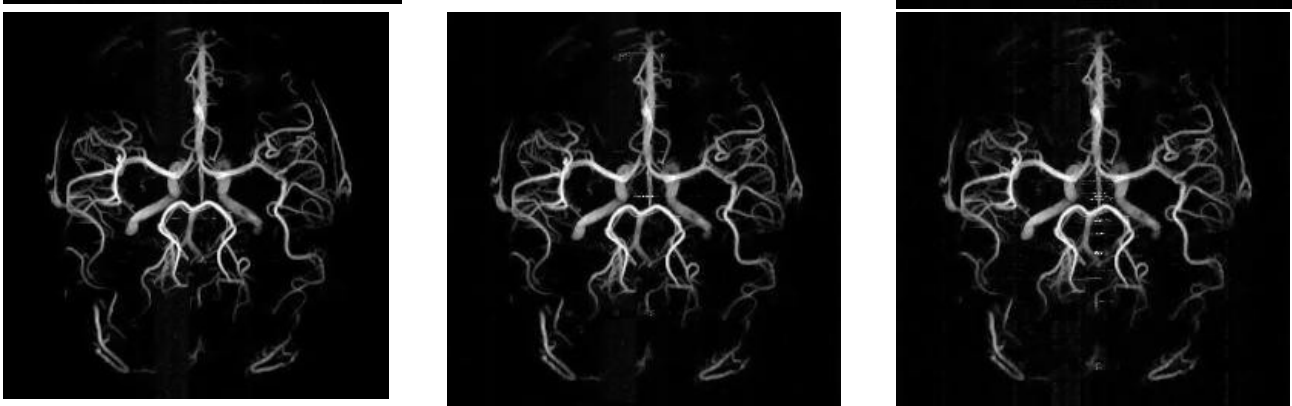

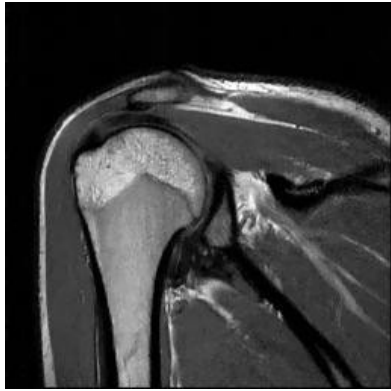

$60 \%$ sampling

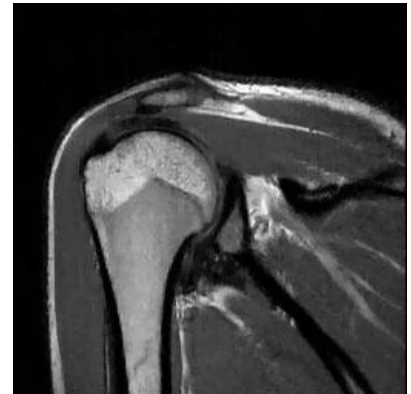

$50 \%$ sampling

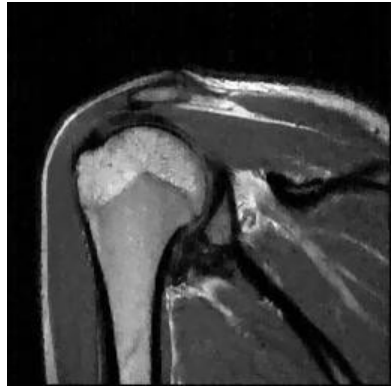

$25 \%$ sampling

Figure 4: CS MRI reconstruction of Brain, Shoulder and Angiogram MR Images with random undersampling pattern with (a) $60 \%$ sampling (b) $50 \%$ sampling (c) $25 \%$ sampling

To show the differences in the quality of reconstruction for Cartesian and random undersampling, PSNR, MSE and SSIM of reconstructed MR image is recorded at different sampling percentages. From Table I, it is observed that random undersampling provides improved result even at lower sampling percentages. The quality of reconstructed MRI in terms of PSNR, MSE and SSIM demonstrates the novelty of the proposed combination of sampling and reconstruction techniques.

Table I: Numerical Analysis for the CS reconstruction of Brain MR Image

\begin{tabular}{|c|c|c|c|c|}
\hline $\begin{array}{c}\text { Sampling } \\
\text { pattern }\end{array}$ & $\begin{array}{c}\text { Sampling } \\
\text { Percentage }\end{array}$ & PSNR & MSE & SSIM \\
\hline Cartesian & 60 & 37.8552 & 10.6553 & 0.9749 \\
\cline { 2 - 5 } & 50 & 36.2132 & 15.5512 & 0.9708 \\
\cline { 2 - 5 } & $\mathbf{2 5}$ & $\mathbf{2 8 . 2 4 2 0}$ & $\mathbf{9 7 . 4 7 1 7}$ & $\mathbf{0 . 8 9 6 9}$ \\
\hline \multirow{3}{*}{ Random } & 60 & 37.9590 & 9.9189 & 0.9752 \\
\cline { 2 - 5 } & 50 & 36.3176 & 14.8971 & 0.9718 \\
\cline { 2 - 6 } & $\mathbf{2 5}$ & $\mathbf{3 3 . 2 6 5 6}$ & $\mathbf{3 0 . 6 5 6 2}$ & $\mathbf{0 . 9 1 6 4}$ \\
\hline
\end{tabular}

$\mathrm{CS}$ reconstruction from random undersampled k-space has much lower MSE than reconstruction from radial and Cartesian undersampling.

At $25 \%$ of random sampling, MSE and SSIM is 30.6552 and 0.9164 respectively for MRI of brain. Faithful image reconstruction is possible with just $25 \%$ of $\mathrm{k}$-space samples. These results exhibit the importance of dense sampling near the center of $\mathrm{k}$-space for reconstruction.

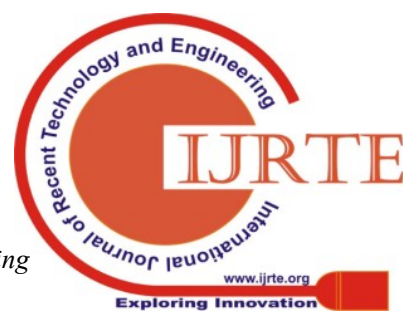


Table II: Comparison of results for three MR images using Radial Sampling

\begin{tabular}{|c|l|l|l|l|}
\hline $\begin{array}{l}\text { Sampling } \\
\text { percentag } \\
\text { e }\end{array}$ & $\begin{array}{l}\text { MR } \\
\text { Image }\end{array}$ & PSNR & MSE & SSIM \\
\hline \multirow{2}{*}{60} & Brain & $\begin{array}{l}37.959 \\
0\end{array}$ & 9.9189 & $\begin{array}{l}0.975 \\
2\end{array}$ \\
\cline { 2 - 5 } & $\begin{array}{l}\text { Head } \\
\text { Angiogra }\end{array}$ & 36.033 & 16.207 & 0.933 \\
& m & 9 & 1 \\
\cline { 2 - 5 } & Shoulder & 33.998 & 25.892 & 0.918 \\
& & 1 & 9 & 0 \\
\hline \multirow{5}{*}{50} & Brain & 36.317 & 14.897 & 0.971 \\
& & 6 & 1 & 8 \\
\cline { 2 - 5 } & Head & 33.904 & 26.460 & 0.899 \\
& Angiogra & 8 & 4 & 8 \\
& m & & & \\
\cline { 2 - 5 } & Shoulder & 32.424 & 37.210 & 0.893 \\
& & 2 & 4 & 2 \\
\hline 25 & Brain & 33.265 & 30.656 & 0.916 \\
& & 6 & 2 & 4 \\
\cline { 2 - 5 } & Head & 31.072 & 47.798 & 0.864 \\
& Angiogra & 3 & 5 & 3 \\
\hline & m & & & \\
\cline { 2 - 5 } & Shoulder & 31.005 & 49.582 & 0.845 \\
& & 8 & 8 & 7 \\
\hline
\end{tabular}

Table II shows the CS MRI reconstruction using Random undersampling pattern for three MR images. The value of SSIM for MRI of brain, shoulder and head angiogram is found to be better even with lesser sampling percentage with appreciable values for PSNR. The ideal value of SSIM is 1.At $25 \%$ of sampling SSIM for brain is 0.9164 , for shoulder is 0.8457 and for head angiogram is 0.8643 . This indicates the structural similarity of the reconstructed image is very close to original MR image. The quantitative and qualitative analysis of the proposed method confirms that the compressive sampling of the k-space does not compromise with the reconstructed image quality, but it speeds up the overall scanning process.

\section{CONCLUSION}

In this paper we proposed the recovery of MR images from the compressively sampled MR k-space using the Particle Swarm Optimization Algorithm by minimizing the fitness function based on MSE of the original and reconstructed image. Total Variation is combined with PSO to reduce the effect of artifacts generated due to k-space undersampling. The random undersampling pattern is applied on MR k-space which collects more samples from the center of the $\mathrm{k}$-space. The proposed combination of sampling and recovery algorithm is applied on different MR images. Sampling the k-space using appropriate undersampling pattern is one of the most important steps in MRI. The Cartesian pattern is simple to construct and also is popular. The experiments conducted prove that the image reconstructed with random undersampling pattern applied on k-space has the better results for PSNR, MSE and SSIM compared to that using Cartesian Sampling trajectory. The proposed method of reconstruction works well in comparison with the standard Cartesian Sampling trajectory used for compressive sampling of k-space.

\section{REFERENCES}

1. Donoho D., "Compressed sensing", IEEE Transaction on Information Theory, Vol. 52, No. 4, pp. 1289-1306, 2006.

2. Candes E.J and Wakin M.B., "An Introduction to Compressive Sampling”, IEEE Signal Processing Magazine, Vol. 25, No. 2, pp. 21-30, 2008.

3. Lustig M., Donoho D. and Pauly J.M., "Sparse MRI: The application of compressed sensing for rapid MR imaging", Magnetic Resonance in Medicine, Vol. 58, pp. 1182-1195, 2007.

4. Sodickson D. K., Mckenzie C. A., "A generalized approach to parallel magnetic resonance imaging", International Journal of Medical Physics, Vol. 28, pp. 1629-1643, 2001.

5. Christopher K. Anand, Paul M. Margosian and Francis H. Bearden, "Two-dimensional phase- conjugate symmetry reconstruction for 3D spin-warp, echo-planar and echovolume magnetic resonance imaging", Philips Medical Systems, 2002.

6. Karla Miller, "Partial k-space Reconstruction", Lecture Notes, FMRIB Centre, Nuffield Department of Clinical Neurosciences, University of Oxford, UK.

7. BarisYuce, Michael S. Packianather, Ernesto Mastrocinque, Duc Truong Pham and Alfredo Lambiase, "Honey Bees Inspired Optimization Method: The Bees Algorithm", International Journal of Insects, vol. 4, pp. 646-662, 2013.

8. Kieren Grant Hollingsworth, "Reducing acquisition time in clinical MRI by data undersampling and compressed sensing reconstruction", Physics in Medicine \& Biology, Vol. 60, pp. 297-322, 2015.

9. Christopher Baker, "Image Comparison of MRI UnderSampling Techniques for Compressed Sensing with Translation Invariant Wavelets Using FastTest CS: A Flexible Simulation Tool", Journal of Signal and Information Processing, Vol. 7, pp. 252-271, 2016.

10. Zhang Lin, "Image Adaptive Recovery Based on Compressive Sensing and Genetic Algorithm", IEEE International Conference on Computer Science and Automation Engineering, pp. 346-349, 2012.

11. J. Kennedy and R. Eberhart, "Particle swarm optimization", Proceedings of IEEE International Conference on Neural Networks, Vol. 4, pp. 1942-1948, 1995.

12. Jawad Ali Shah, Qureshi I.M., Amir A. Khaliq and Hammad Omer, "Sparse Signal Recovery based on Hybrid Genetic Algorithm", Research Journal of Recent Sciences, Vol. 3, No. 5, pp. 86-93, 2014.

13. Dian PalupiRini, Siti Mariyam Shamsuddin and Siti Sophiyati Yuhaniz "Particle Swarm Optimization: Technique, System and Challenges", International Journal of Computer Applications, Vol. 14, 2011.

14. Shiqian Ma, Watao Yin and AmitChakraborty, "An Efficient Algorithm for Compressed MR Imaging using Total Variation and Wavelets", IEEE Conference on Computer Vision and Pattern Recognition, 2008.

15. Tanuj Kumar Jhamb, Vinith Rejathalal and V.K. Govindan, "A Review on Image Reconstruction through MRI k-Space Data", International Journal of Image, Graphics and Signal Processing, Vol. 7, pp. 42-59, 2015. 